\title{
X-Ray Lasers
}

\author{
P. Jaeglé, Orsay
}

(Lab. de Spectroscopie Atomique et lonique, Université Paris-Sud)

Recent progress leads us to believe that X-ray lasers will, in the near future, become generally available as a new tool for science and technology. Their output is in the soft $X$-ray range, the shortest wavelengths attained being a few nanometers. In this range, they will be by far the most brilliant and coherent sources with applications in such different fields as living sample holography, contact microscopy, lithography, surface studies, flash spectroscopy and so on.

Lasing, as is well known, arises from stimulated photon emission whereby the radiative decay of an excited system is induced by an incident photon instead of occurring spontaneously. This is the key to getting amplification. It is also well known that the Einstein coefficients $A$ and $B$ defining respectively the probabilities of spontaneous and stimulated emission are related by the following:

$$
A=\left(8 \pi h / \lambda^{3}\right) B
$$

where $\lambda$ is the wavelength. For a long time it was thought that this implied that X-ray lasers were an impossibility since the spontaneous emission rose so dramatically with wavelength. It was not appreciated that the intensity of emission from a macroscopic medium was not proportional to $A$ or $B$, except in very dilute gases. To derive the macroscopic intensity, it is necessary to take account of radiative transfer, which in the simplest case can be expressed as:

$$
I=S(1-\exp -k L)
$$

where $S$ is a source function independent of $A$ and $B$, and $k$, which involves both absorption and stimulated emission, has the form:

$$
k=(h g B / \lambda) \triangle N F(\lambda) \text {. }
$$

$\triangle N$ is the reduced population difference (i.e. divided by a statistical weighting $g$ ) between the lower and the upper level of the transition under consideration and $F(\lambda)$, a profile function. Seeing these expressions, it becomes clear that the relevant parameter for lasing action is not $\lambda$ but $\Delta N$ because, whatever the value of $\lambda$, if there exists a population inversion in the medium, $\Delta N$ becomes negative and $k$ turns into an amplification instead of an absorption coefficient. This holds for $\mathrm{X}$-rays as well as much longer radiation.
The true significance of the scaling law involved in expression (1) appears in the pumping power needed to achieve population inversion. It is much larger for $\mathrm{X}$-rays than for longer wavelengths. Not only must the transition energy be larger than for, say, visible light, but the time allowed for population inversion is considerably reduced, since the radiative life-time of the upper level decreases with the third power of the wavelength. That is why it is extremely difficult to imagine an efficient laboratory system which uses pure $\mathrm{X}$-ray pumping. Taking into account the efficiency of X-ray sources and the low absorption of the lasant, the required power would have to come from a nuclear explosion rather than from any laboratory source!

\section{Population Inversion}

The important discovery of the last ten years has been the fact that population inversions can occur sometimes spontaneously in hot dense plasmas produced by the powerful lasers used in thermonuclear fusion research. This has been observed in several laboratories: at Hull University and at Rutherford Laboratory in the UK, at Livermore Laboratory and at Princeton University in the USA, at GRECO "Interaction Laser-Matière" (Palaiseau)* and Centre d'Etudes de Limeil in France, and at Osaka in Japan [1, 2, 3].

Two types of mechanism have been identified as the main sources of population inversion. Relatively simple to understand is the recombination process which arises from the rapid cooling of the plasma causing a large departure from thermodynamic equilibrium, and leading to transient population inversions between some of the excited levels of plasma multicharged ions. Less evident is the mechanism that relates to the specific atomic properties of peculiar ion species in the so-called collisional scheme. Here population inversions are produced as a result of a balancing between the excitation of the ground state species, induced by collisions with

* now LULI, the National Facility for Use of Intense Lasers of CNRS and Ecole Polytechnique.

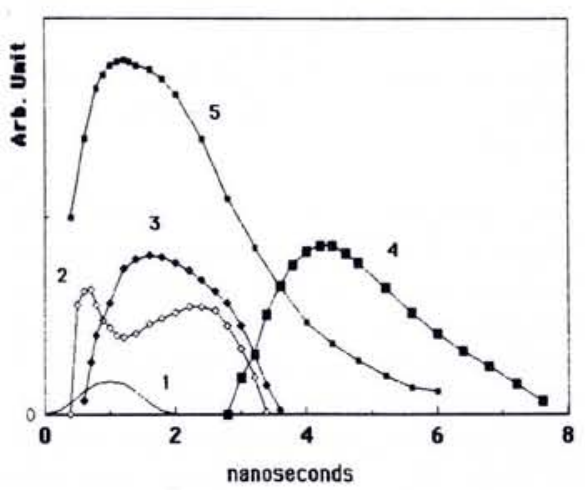

Fig. 1 - Recombination pumping.

(1) Nd-laser pulse;

(2) density of ions of charge $z$;

(3) density of ions of charge $z+1$;

(4) density of population inversion between two levels of $z$ ions;

(5) plasma temperature. Population inversions appear between levels of different main quantum number, $(\Delta n \neq 0)$.

free electrons, and the fast radiative decay of low lying levels.

The qualitative features of the recombination scheme are presented in Fig. 1, which displays the temporal variation of various parameters of a plasma produced by a laser beam focussed onto the surface of a solid target. The laser pulse (curve 1 ) is assumed to have a width of about 2 ns. Plasma temperature is represented by curve 5 where an order of magnitude for the maximum is $200 \mathrm{ev}$ $(1800000 \mathrm{~K})$. Plasma ionization appears in curves 2 and 3 which show the density variation of two successive ions, i.e. ions of the same target element but differing from each other by 1 unit of electric charge, say $z$ and $(z+1)$ ions. The density of population inversion,

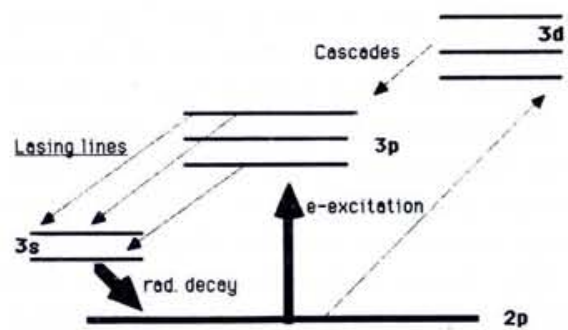

Fig. 2 - Collisional pumping. Excitation of the ground level by free electron collisions populates the $3 p$ levels directly or via the $3 d$ levels. Population inversions appear between levels $3 p$ and $3 s,(\Delta n=0)$. 


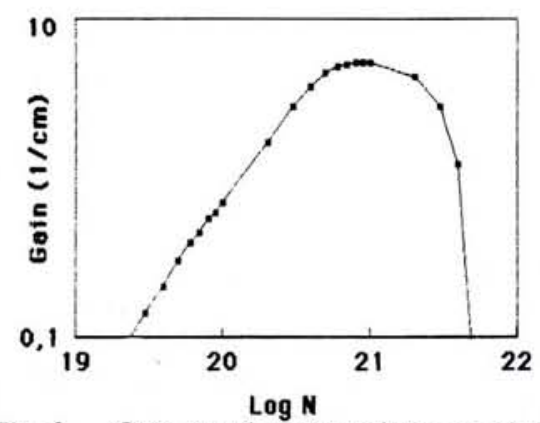

Fig. 3 - Gain as a function of plasma electronic density at fixed temperature in $\mathrm{Ne}$ like ions.

$\Delta N$, between two given electronic levels of the less charged ion (z-charge) is displayed in curve 4. Plasma electronic density, which is not represented in this figure, has a maximum near $10^{23}$ $\mathrm{cm}^{-3}$ but it is no larger than $10^{19} \mathrm{~cm}^{-3}$ or less during population inversion. As can be seen by numerical simulation, the population ratio $(z+1)$ ion/ $z$ ion, during plasma cooling, is much larger than it would be at equilibrium. Then population inversions are provoked by the recombination of $(z+1)$ ions with free electrons which populates the highlying levels of $z$ ions more rapidly than the low-lying ones. Simultaneously, the lower levels have a faster radiative decay to the ground level. Curve 4 in Fig. 1 shows some important characteristics of population inversions produced by recombination: they are delayed with respect to the laser pulse and they occur at relatively low temperature in the plasma. Moreover these population inversions occur between levels with different principal quantum numbers $(\Delta n \neq 0)$, for instance 2 and 3 in hydrogenic ions and 3 and 5 or 4 in lithiumlike ions. The range of inversion duration is from one to a few nanoseconds.

Turning now to the collisional sche$\mathrm{me}$, the production of population inversion is purely a matter of transition rate balance in neon-like or nickel-like ions. Let us look at the Ne-like $2 p^{6}$ ground level (Fig. 2). In round terms, the larger the plasma electronic density the larger will be the collisional excitation rate from the ground level to $2 p^{5} 3 p$ levels. Nevertheless, the radiative decay probability remains constant for all levels, including that for the $2 p^{5} 3 s$. This leads to the appearance of population inversions between $3 p$ and $3 s$ levels when the electronic density becomes larger than some minimum value. The gain curve of Fig. 3 illustrates this behaviour at fixed plasma temperature. The numbers show the correct order of magnitude rather than values exactly calculated for a well defined transition. The gain starts at $2-3 \times 10^{19} \mathrm{~cm}^{-3}$ and reaches a maximum around $5 \times 10^{20}$ $\mathrm{cm}^{-3}$. Then the increasing collisional rate of all transitions begins to reduce and finally the population inversion is destroyed.

The detailed study of the process, especially the role of cascades from $3 d$ to $3 p$ levels as well as dielectronic recombination, needs a great deal of quantum mechanical calculation. Numerical modelling may include several hundred population equations. In contrast to recombining plasma, population inversions occur during the laser pulse and at high temperature (typically $800 \mathrm{ev}$ ). The duration is a few hundreds of picoseconds only. For inversions to take place between levels with the same principal quantum number $(3$ for $\mathrm{Ne}$ like, 4 for $\mathrm{Ni}$-like ions), $z$ must be larger than in recombination systems at equal lasing wavelength.

\section{Amplification}

Once a method of generating population inversion is known, amplification can be looked for in plasma columns, whose length is sufficient to satisfy equation (2), which we rewrite as:

$$
I=S^{\prime}(\exp G L-1)
$$

where $S^{\prime}=-S$, and $G=-K$.

This ensures that $I$ is largely dominated by stimulated emission. A requirement is to focus the laser beams using cylindrical lenses. The principle of producing the amplifying medium is shown in the sketch of Fig. 4. Amplification occurs along the column axis, but only in the plasma region where the density and temperature are suitable for generating the ion species able to give rise to population inversion. These features of the plasma are presented on the left of the figure, but it must be kept in mind that these parameters are changing rapidly with time.

Two examples of experimental arrangements able to achieve plasma lengths of $5 \mathrm{~cm}$ and $6 \mathrm{~cm}$ respectively are shown in Figs 5 and 6 . The first has been built at Livermore (USA) near the very big Nova Laser, the beams of which are $74 \mathrm{~cm}$ in diameter and supply an energy of several $\mathrm{kJ}$, at $\lambda=0.53 \mu \mathrm{m}$, on both sides of the thin foil target. The second is at Palaiseau using the laser of LULI. Its single side illumination has been designed for targets deposited on massive supports. Each beam is $9 \mathrm{~cm}$ diameter and carries 100-150 $\mathrm{J}(\lambda=$ $1.06 \mu \mathrm{m})$. At the Rutherford Laboratory a thin fibre target technique, allowing column lengths up to $12 \mathrm{~mm}$, has been developed. At Princeton (USA), a magnetic confinement device increases the population inversion by maintaining a high density during plasma cooling.

\section{Progress Towards a Device}

Knowing how to amplify the radiation, where are we in the matter of X-ray laser building? A gain-length product (GL) of 15, the highest ever achieved, has been obtained at Livermore, at a wavelength of $206 \AA$ in neon-like selenium. This should be nearly sufficient to reach a strongly saturated regime. The brightness of the source, is such that the device can be considered to be working as a laser, but because of plasma inhomogeneities and the absence of an optical style cavity, the spatial coherence of the radiation is still at the level of common X-ray sources. At Princeton, where hydrogenic carbon in recombining plasma has been investigated with the help of magnetic confinement, a $G L$ value of 8 at $182 \AA$ wavelength has been reported and the directivity of the beam seems to be quite good. Lithiumlike aluminium, investigated at Palaiseau with comparatively cheap means, does amplify radiation at $105.7 \AA$ but, at the present stage of the work, the $G L$ does not exceed a value of about 4 .

A great step towards lasers of good quality will be made when optical cavities are used. For instance, at Palaiseau even a 4- or 6-pass cavity would lead to very high brightness as well as to high

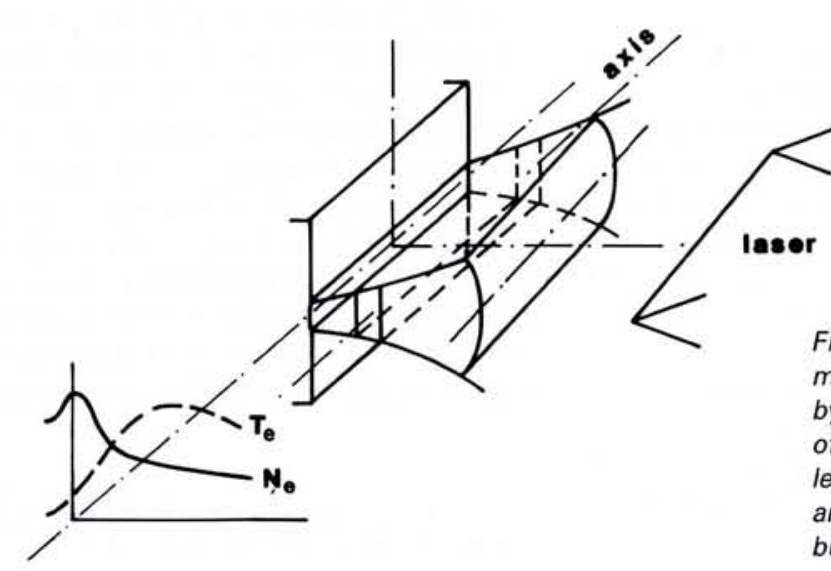

Fig. 4 - Sketch of plasma column production by cylindrical focussing of laser beam. On the left, electronic density and temperature distribution at a given time. 


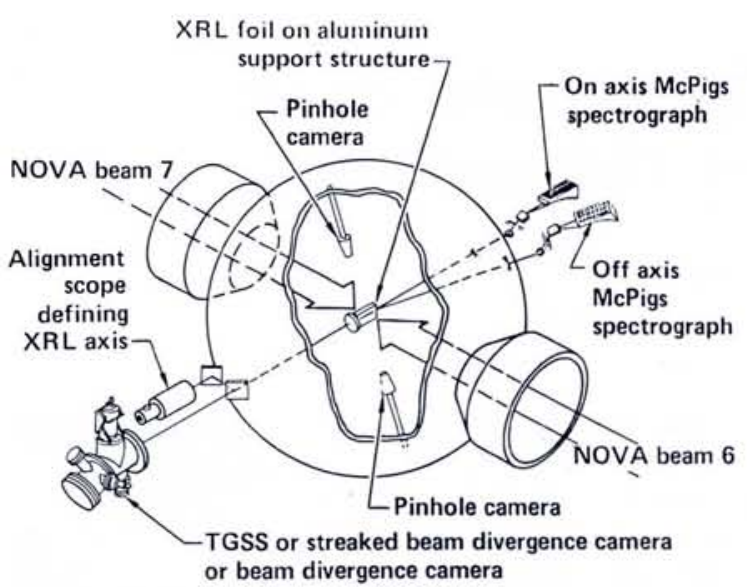

Fig. 5 - Experimental chamber and main diagnostics at Livermore. The diameter of the NOVR laser beams is $74 \mathrm{~cm}$. The $X$-ray gain is measured by the "on axis McPigs spectrogtraph". Plasma length: $5 \mathrm{~cm}$.

beam coherence. Although preliminary experiments have already demonstrated the efficiency of multilayer mirrors for soft X-ray lasers, two kinds of difficulty have still to be overcome for cavities to come into current use. The technique for making the mirrors is difficult and, even if a $10 \%-20 \%$ reflection coefficient can be achieved, it is still a problem to control accurately the wavelength of the maximum of the reflection band. However one can reasonably think that substantial progress will be made in these matters in the coming years.

For the cavity actually to work, it is necessary that the duration of popula- tion inversion in the plasma is much longer than the light flight-time between the mirrors. For a typical cavity length of $10 \mathrm{~cm}$, this time $\cong 300$ ps. Remembering the characteristics mentioned above, it is clear that this condition is far from being always satisfied, especially in the collisional scheme. Progress has still to be made either in increasing the gain duration in the case of collisional pumping or in increasing the gain coefficient in the case of longpulse recombination pumping. This could entail new requirements, not only for target designs, but also the characteristics of the lasers which are used for plasma production.

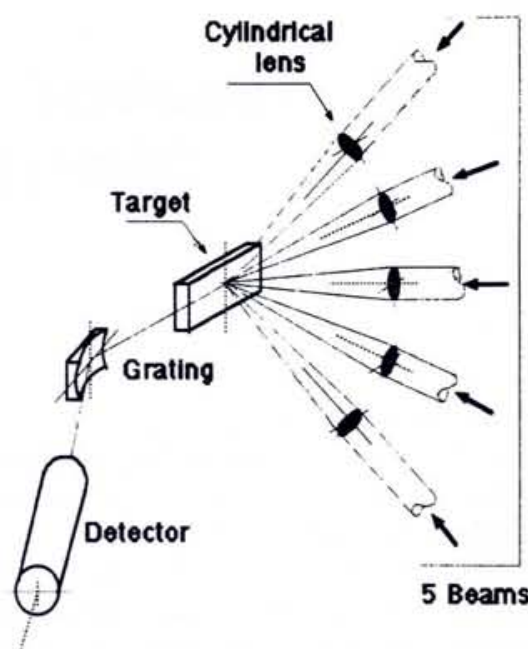

Fig. 6 - Sketch of the experiment of Palaiseau, where massive target support is made possible by using single side illumination. Plasma length: $6 \mathrm{~cm}$.

\section{REFERENCES}

[1] 'Proceedings of International Colloquium on X-ray Lasers', eds. P. Jaeglé and A. Sureau, Journal de Physique 47, Colloque C6, Supplement No. 10, 1986.

[2] 'The Generation of Coherent XUV and Soft-X-Ray Radiation', eds. D.L. Matthews and R.R. Freeman, J. Opt. Soc. Amer., 4 (1987) feature edition.

[3] 'Short Wavelength Lasers and Their Applications', ed. C. Yamanaka, Proceedings in Physics 30 (Springer-Verlag) 1988.

\section{CATEGORY 4a)}

G. Benincasa, CERN, Geneva M.A. Crusellas,

Sta. Eulalia de Riuprimer, E P.-A. Nilsson, Göteborg, S

J. Wagner, Würzburg, D

R. Zangara, Palermo, I

\section{CATEGORY 4c)}

Austrian Physical Society

J. Geisselbrecht, Zell am See

Belgian Physical Society

J. Vanregenmorter, Brussels

C.J.M. Vlekken, Leuven

Eötvös Lorand Physical Society

F. Csikor, Budapest

Finnish Physical Society

J.T. Puustinen, Helsinki

M. Rinta-Nikkola, Jyväskylä

French Physical Society

M. Barat, Orsay

B. Buffat, Aubervilliers

D. Challeton, Grenoble

P.A. Chenevas, Rives-sur-Fure

H. Dreysse, Vandœuvre-Nancy

E. Molva, Grenoble

J.C. Villegier, Grenoble

\section{New Members of the European Physical Society}

German Physical Society

K.-W. Hasselbach, Grenoble, F

R. Hereth, Essen

J. Kircher, Stuttgart

M. Klaey, Neuhaus, $\mathrm{CH}$

J. Kübler, Darmstadt

H. Morgner, Witten

T. Müller, Grenoble, $F$

C.-S. Neumann, Winnenden

W. Schomburg, Bochum

P. Stichel, Bielefeld

M. Treichel, Neuchâtel, $\mathrm{CH}$

C.A.Z. Vasconcellos, Erlangen

M. Warns, Bonn

Hellenic Physical Society

P. Anagnostakis, Alimos

E.C. Paloura, Thessaloniki

The Institute of Physics

J.-P. Connerade, London

Italian Physical Society

C.E. Agnes, Turin

G. Agosti, Wettingen, $\mathrm{CH}$

A. Borsellino, Trieste

G. Furlan, Trieste

C. Mariani, Modena

L. Reatto, Milan

The Netherlands' Physical Society R.A. de Groot, Nijmegen

H. Dijkerman, Vleuten
Israel Physical Society

D. Horn, Tel-Aviv

Physical Section, Union of Czechoslovak Mathematicians and Physicists

L. Pust, Prague

H. Stikova, Prague

B. Velicky, Prague

Physical Section, Union of Yugoslav Societies of Mathematicians, Physicists and Astronomers

S. Milosevic, Zagreb

S. Pesic, Belgrade

Physical Society of the German Democratic Republic H.P. Friedrich, Berlin K. Henneberger, Berlin

G.O. Müller, Berlin

D. Geiss, Teltow

H. Gündel, Berlin

Polish Physical Society

P. Chankowski, Warsaw

A.W. Domanski, Warsaw

W. Florkowski, Krakow

K. Golec-Biernat, Krakow

A. Horzela, Krakow

M. Kutschera, Krakow

O.W. Morawski, Warsaw

J. Sworakowski, Wroclaw

K. Zalewski, Krakow
Romanian National Committee for Physics

A.Gh. Podoleanu, Bucharest

Spanish Royal Society of Physics J.M. Ugalde, Donostia

Swiss Physical Society

M.M. Freund, Zurich

M.-O. Hongler, Nyon

J. Lister, Pully

\section{CATEGORY 4d)}

American Physical Society

F. Azgui, Alger, Algeria

L. Mihaly, Stony Brook, NY

J. Missimer, Villigen, $\mathrm{CH}$

M. Haruki, Zurich, $\mathrm{CH}$

Physical Society of Japan

T. Kimura, Ibaraki

T. Murayama, Tokyo

French Optical Society

C. Cavaillier, Courtry

M. Colombier, Clermont Ferrand

J.P. Pocholle, Orsay

Spanish Optical Society

R. Corbalan, Sant Cugat del Valles 